\title{
Enhancement of medical student perception of surgical specialization with peer-assisted laparoscopic simulation: a pilot study
}

\section{Jordan J. Baechle, ${ }^{a}, *$ Alexander M. Lopez, ${ }^{a}$ Jyotsna Thota, ${ }^{a}$ Mitchell F. Bowers, ${ }^{a}$ Paula Marincola Smith ${ }^{b}$ and Regina S. Offodile ${ }^{a, c}$}

${ }^{a}$ School of Medicine, Meharry Medical College, Nashville, TN, USA; ${ }^{b}$ Department of Surgery, Vanderbilt University Medical Center, Nashville, TN, USA; ${ }^{c}$ Department of Clinical Skills and Competencies, Meharry Medical College, Nashville, TN, USA

${ }^{*}$ Corresponding author at: School of Medicine, Meharry Medical College, Nashville, TN, USA. Email: jbaechle18@email.mmc.edu

Date accepted for publication: 19 August 2020

\section{Abstract}

Background: Although the pre-clinical years are pivotal in career path commitment for medical students, this phase of education is traditionally devoid of surgical exposure. Although peer-assisted learning and simulation are both validated methods of supplementing early exposure to medical specialties, their use in familiarizing students with modern surgical procedures remains minimal. We hypothesize that student-led laparoscopic simulation will result in significantly increased self-perception of surgical clerkship preparedness and confidence in pursuing surgical residency among pre-clinical medical students. Methods: Laparoscopic simulation workshops were organized by student educators who underwent faculty-led leadership training in simulation education. Pre-clinical medical students were invited to participate in weekly 1-hour laparoscopic simulation events that progressed through a series of coordination exercises using box trainers over the course of 8 weeks. Participants completed pre- and post-simulation surveys concerning perceptions toward surgical clerkship and residency. The results of these questionnaires were analyzed using the Wilcoxon Mann-Whitney test. Results: Among the 28 participants, 9 (32\%) were first-year pre-clinical medical student and $19(68 \%)$ were second-year medical students. Participants attended an average of 2.8 of the 8 sessions. On a 100point scale, mean student perceptions of surgical clerkship preparedness and confidence pursuing surgical residency scored 44.8 and 50.3, respectively. Upon completion of the post-test, these mean preparedness and confidence scores were significantly increased to 58.6 and 66.6 , respectively $(P<0.001)$. Conclusion: Peer-assisted laparoscopic simulation learning may be an effective means of enhancing pre-clinical student perceptions toward surgical fields in medical school curricula, however, longitudinal studies are needed to assess the impact on surgical clerkship proficiency and residency matriculation/completion.

Keywords: student; simulation; surgery; laparoscopic; peer-assisted learning

\section{Introduction}

Medical students experience a dearth of exposure to the surgical fields compared with other medical specialties in their pre-clinical years. ${ }^{1,2}$ Despite statements from the American Surgical Association suggesting more surgical exposure earlier in medical education, pre-clerkship surgical education remains minimal in the United States compared with other specialties. ${ }^{3}$ In a 2013 study surveying 128 US medical schools, only $22 \%$ of the respondents reported any implementation of elective pre-clinical surgical skills workshops and only 36\% reported offering any surgical exposure into the first-year curriculum. ${ }^{4}$

As health care becomes increasingly specialized and residency training programs follow suit by offering tracks in early specialization, students have been encouraged to commit to their career trajectory earlier in their education to be competitive applicants when applying for residency. ${ }^{5,6}$ This hyper-specialization of the field and lack of surgical exposure in the preclinical years has likely discouraged pre-clinical students by fostering a sense of unpreparedness and lack of confidence. ${ }^{5}$ It

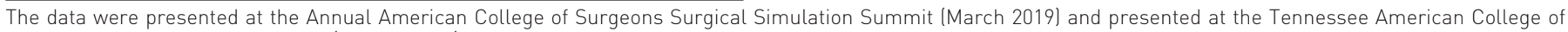
Surgeons annual chapter meeting (August 2019). 
has been reported that as many as 59\% of medical students choose their specialization during their pre-clinical years, ${ }^{7}$ and that simulation exercises during the pre-clinical years are highly influential, particularly in surgery. ${ }^{6-10}$ Student interest groups are often organized to supplement early exposures to medical specialties utilizing peer-assisted learning (PAL). ${ }^{11,13}$

PAL is a well-established teaching concept, used by many institutions, that uses teaching sessions organized by students for students. ${ }^{13}$ The benefits of peer-assisted over instructorled learning include providing a low-pressure learning environment while relieving the time burden on faculty. ${ }^{12,13}$ Studies have demonstrated the use of PAL simulations in enhancing student education and exposure in pediatrics, ${ }^{14}$ emergency medicine, ${ }^{15,16}$ neurology, ${ }^{17}$ obstetrics, ${ }^{18}$ and clinical anatomy. ${ }^{19} \mathrm{PAL}$ in surgical education has been shown to be instrumental in the transferring suturing skills from student to student, ${ }^{20}$ however the impact of laparoscopic simulation in medical school education has been minimal.

The purpose of this study was to assess the efficacy of PAL on student perceptions toward a surgical career through student-led laparoscopic simulation sessions. We hypothesize that offering student-led laparoscopic simulation sessions to pre-clinical medical students will result in significantly improved self-perception of preparedness for the surgical clerkship as well as increased confidence in pursuing a surgical residency.

\section{Methods}

\section{Peer-assisted learning}

Laparoscopic simulation workshops were organized by qualified student coordinators who underwent faculty-led simulation training. Student educators assembled a debriefing and itinerary before each event. The debriefing consisted of an outline of each event's laparoscopic coordination task and a 5-minute demonstration video. The workshop introduction lasted 15 minutes, giving the event a 3:1 practical-to-didactic ratio in time utilization. The demonstration video was sent to each registered participant 3 days before the event. During each event, two students were assigned to one trainer box and alternated at 10-minute intervals to attempt the predefined task. Participants progressed serially through an adapted Fundamentals of Laparoscopic Surgery (FLS) curriculum ${ }^{21}$ (Fig. 1). The specific task rubrics are listed in the Appendix. These sessions ran once a week for 1 hour over the course of 8 weeks.

\section{Data collection}

Institutional Review Board (IRB) approval was obtained from Meharry Medical College, Nashville, for this study (IRB\# FWA00003675). All pre-clinical medical students attending Meharry Medical College were invited to participate in this study on a first come first served basis. Before participation, informed consent was granted by all student participants. A REDCap (Research Electronic Data Capture) survey was developed to quantify the pre- and post-simulation series intervention confidence and preparedness on a 100-point scale (0 not confident at all, 100 fully confident; 0 completely unprepared, 100 fully prepared). The survey was distributed electronically, and participants were asked to complete an anonymous survey at the beginning and end of the 8-week course that asked them to quantify their sense of preparedness for the surgical clerkship and their
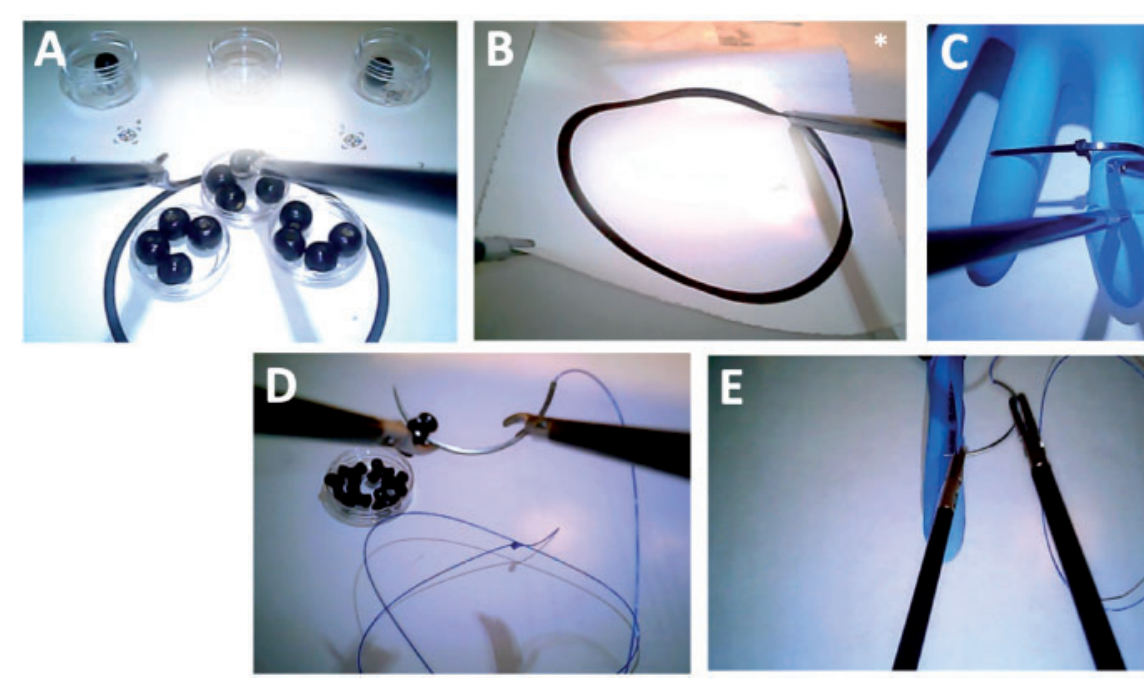

Figure 1. Adapted laparoscopic fundamentals exercises. A series of five tasks adapted from the FLS certification examination: (A) ball transfer, (B) precision cutting*, (C) zip-tie ligation, (D) needle threading, and (E) suture and knot tying. *Omitted if cutting instrument unavailable. 
confidence level in pursuing surgical residency on a 100point scale. Data were analyzed using SPSS software (version 25, IBM). Descriptive and nonparametric testing was performed due to the relatively small sample size and a Wilcoxon Mann-Whitney test to assess changes in student perceptions.

\section{Results}

Twenty-eight pre-clinical medical students attended at least one student-led surgical simulation exercise. The participant demographics are summarized in Table 1. Sixteen (57\%) were male and $12(43 \%)$ were female. The median age of the participants was 24.5 years. Nine (32\%) were first-year medical students and 19 (68\%) were second-year students. Among the student participants, 27 (96\%) had heard of laparoscopic surgery, 2 (7\%) participants had observed a laparoscopic operation, and 1 (4\%) had previously used a laparoscopic training device before study enrollment. Before the simulation workshops, 6 (21\%) participants reported being particularly interested in pursuing a surgical career and $12(43 \%)$ were unsure.

Within the 8-week period, students attended an average of 2.8 sessions (range, 1-7). After the simulation workshops, $14(50 \%)$ participants reported being particularly interested in pursuing a surgical career and 11 (39\%) were unsure (Table 2). Among all participants, there was a significant increase in both self-reported preparedness for the surgical clerkship (mean, 44.8 vs 58.6; $P<0.001$ ) and confidence in pursuing a surgical residency (mean, 50.3 vs 66.6; $P<$ 0.001) (Figs 2 and 3) after completion of the PAL laparoscopic simulation curriculum.

In addition, 27 (96.4\%) of the participants reported that the student-led simulation sessions had a positive impact on their perception of surgery, and $26(92.9 \%)$ responded that the workshops enhanced their interest in the field. Twentyfour $(85.7 \%)$ participants felt that student-led simulation sessions had the potential to supplement the pre-clinical medical school curriculum to compensate for an otherwise lack of exposure to surgical disciplines (Table 2).

\section{Discussion}

In this study, we demonstrated an increase in self-perceived preparedness for the surgical clerkship and improved confidence in pursuing a surgical residency among pre-clinical medical students after completion of a peer-assisted laparoscopic simulation curriculum. These results are consistent with the previously published reports that PAL and
Table 1. Participant demographics and pre-simulation interests and experiences

\begin{tabular}{ll}
\hline Variable & Number (\%) \\
\hline Total & $28(100)$ \\
Student year & $9(32)$ \\
First year & $19(68)$ \\
Second year & \\
Gender & $16(57)$ \\
Male & $12(43)$ \\
Female & $19(68)$ \\
Race & $6(21)$ \\
Black & $3(11)$ \\
White & $24.5(25.9 ; 23.2)$ \\
Other & \\
Age (years), median (IQR)
\end{tabular}

simulation are beneficial in medical school education, ${ }^{8}$ particularly in the pre-clinical setting.

Although previous studies have demonstrated that workshops focusing on laparoscopic skills enhance student proficiency in the operating room, ${ }^{22,23}$ this is the first study to examine how such PAL in this context may impinge on student confidence and interest. In our study, over $90 \%$ of participants reported that peer-assisted laparoscopic simulation had a positive impact on their perception of surgery and that their interest in the field was enhanced by the experience. Furthermore, $86 \%$ of participants felt that PAL may be a beneficial supplement to the pre-clinical medical school curriculum and the number of students interested in surgery increased by more than two-fold after the PAL laparoscopic simulation series.

Previous studies have shown a similar increase in pre-clinical student interest after faculty-led surgery skills boot camps; however, these studies routinely acknowledge the burden of faculty time as well as the use of expensive equipment. ${ }^{24}$ Due to the high cost of more advanced modules, student surgery interest groups have traditionally been limited to suturing and knot tying workshops. ${ }^{25-28}$ We believe that our study is impactful, producing a comparable increase in surgical perceptions by utilizing low-cost materials and minimal faculty involvement. This can be critical considering cost and faculty time can both be primary obstacles in the implementation of surgical simulation curricula. $^{29}$

We acknowledge this study carries several limitations. First, inherent volunteer sampling biases exist in the nature of this 
Table 2. Participant perceptions, interests, and experiences

\begin{tabular}{|c|c|c|c|c|c|c|}
\hline \multirow[t]{2}{*}{ Variable } & \multicolumn{3}{|c|}{ Pre-simulation, $\mathbf{n}(\%)$} & \multicolumn{3}{|c|}{ Post-simulation, n (\%) } \\
\hline & Yes & Unsure & No & Yes & Unsure & No \\
\hline Have you heard of laparoscopic surgery before the invitation for this event? & $27(96)$ & $0(0)$ & $1(4)$ & $\mathrm{N} / \mathrm{A}$ & N/A & N/A \\
\hline Have you previously observed a laparoscopic surgery? & $2(7)$ & $0(0)$ & $26(93)$ & N/A & N/A & N/A \\
\hline Have you previously used a laparoscopic training device? & $1(4)$ & $0(0)$ & $27(96)$ & $\mathrm{N} / \mathrm{A}$ & N/A & N/A \\
\hline Are you interested in pursuing a surgical career? & $6(21)$ & $12(43)$ & $10(36)$ & $14(50)$ & $11(39)$ & $3(11)$ \\
\hline $\begin{array}{l}\text { Did student-led laparoscopic simulations have a positive impact on } \\
\text { your perception of surgery? }\end{array}$ & NA & NA & NA & $27(96)$ & $1(4)$ & $0(0)$ \\
\hline Did student-led simulations enhance your interest in the field of surgery? & NA & NA & NA & $26(93)$ & $2(7)$ & $0(0)$ \\
\hline Does early exposure to laparoscopy improve your understanding of surgery? & NA & NA & NA & $26(93)$ & $2(7)$ & $0(0)$ \\
\hline $\begin{array}{l}\text { Do you feel student-led simulations may supplement the lack of surgical } \\
\text { exposure on pre-clinical curricula? }\end{array}$ & NA & NA & NA & $24(86)$ & $4(14)$ & $0(0)$ \\
\hline
\end{tabular}

NA, not applicable.

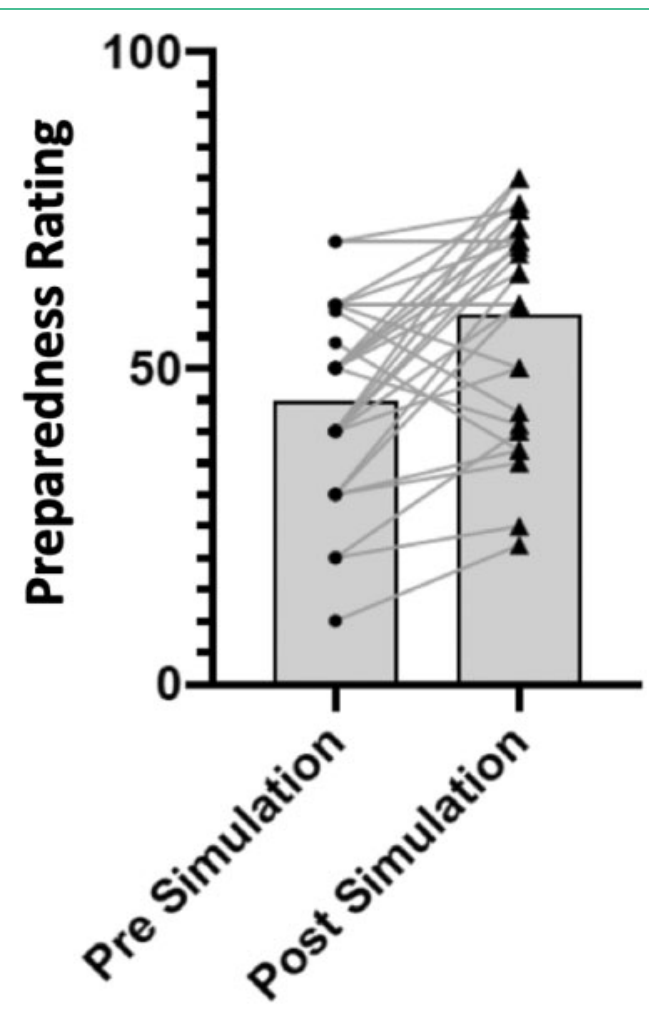

Figure 2. Self-perception of surgery clerkship preparedness.

single-institution study, which limits our ability to generalize our conclusions. The volunteer basis for study accrual inherently selects those students most interested and/or open to pursuing a career in surgery, which may also limit the generalizability of our findings. Notably, however, the initial preparedness and confidence polls suggest even this cohort of volunteer students was self-perceived as

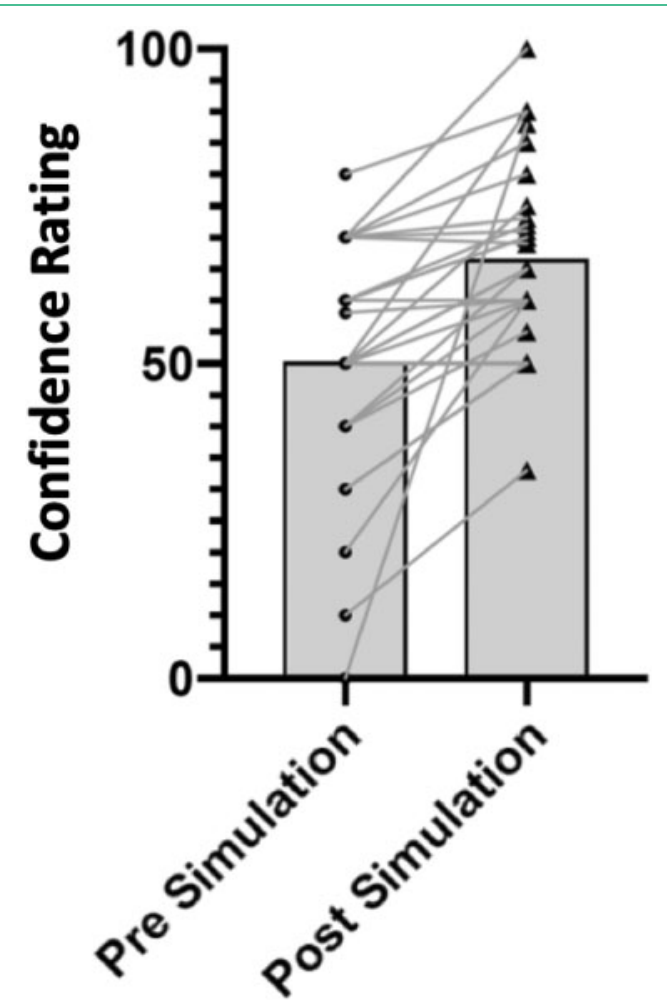

Figure 3. Self-perception of confidence toward surgical residency.

relatively unprepared for surgical clerkship and only slightly confident toward pursing a surgical residence. Furthermore, the pre- and post- surveys were administered to the same cohort before and after the PAL laparoscopic simulation workshop, ensuring the pre- and post-simulation groups were balanced in demographics and previous experiences. Second, this study is observational in the sense that it 
lacks a control group, thus limiting our ability to definitively attribute changes in student perception to our PAL sessions aside from changes attributed to other factors (such as the standard medical school curriculum). However, no other surgery-related curricular events, electives, or skills workshops were made available to pre-clinical students at the institution during the study period. Although low-cost adaptations of the FLS program have been validated in other studies, skills assessment was not the focus of this study, and the series of coordination tasks were adapted with available resources to provide a range of engaging exercises and a learning environment. We therefore cannot validate the efficacy in skill training of the trainers and tasks. However, we highlight student PAL in laparoscopy skills as an area for future studies, because previous research has suggested early exposure to laparoscopic coordination exercises is associated with advanced camera skills and expedited skills progression in residency. ${ }^{29,30}$ Other areas of future study could also include comparative studies on student perceptions after simulation and didactic surgical exposure, longitudinal studies to examine the impact of surgical simulation and its impact on surgical residency matriculation, as well as skills progression studies and how skills progression may correlate with self-perceived confidence and preparedness and the cost and time efficiencies of such interventions.

Despite these limitations, our study utilizes PAL simulations designed to extend the conventional hospital teaching hierarchy to include pre-clinical students by utilizing seniorstudent liaisons and low-cost simulation exercises. It is reasonable to wonder whether unfamiliarity with surgical practice and equipment may deter some students from fully engaging on their surgical clerkships who might otherwise succeed in the field. Such unease is likely to foster uncertainty in clerkship preparedness for pre-clinical medical students and contribute to low confidence levels in their ability to succeed in surgical residency. In addition to the unfamiliarity of surgery, lifestyle factors (including hospital hours, call schedule, and residency length) are also commonly cited deterrents for pre-clinical students (particularly among female students contemplating work-life balance). ${ }^{31-33} \mathrm{At}$ the 2016 Association of Surgeons in Training annual conference, $91 \%$ of medical student respondents agreed that a better balance of training and service within worked hours would improve their perceptions of surgery. ${ }^{34}$

Lastly, increasing a student's sense of preparedness and confidence could indirectly support medical student matriculation into surgical disciplines. Students with fostered interest, heightened confidence, and perceived preparedness may be more likely to engage in other surgery-related educational opportunities, such as joining student interest groups, participating in journal clubs, attending conferences, or seeking out mentorship in the field. Although gender and racial diversity in medical school matriculation has greatly improved over the past decades, ${ }^{35}$ such a trend has been slow to translate to surgery. ${ }^{36,37}$ Engaging students in their pre-clinical years with interactive simulation workshops could broaden the pipeline toward surgical fields among historically under-represented groups, although such a hypothesis would need to be examined prospectively.

In summary, our study showed that participating in a peerassisted laparoscopic simulation curriculum was associated with significantly enhanced pre-clinical student self-perception of preparedness for the surgical clerkship and confidence in pursuing a surgical residency. We believe this method to be cost effective and efficacious, and we invite other institutions to validate our findings by implementing programs that enhance exposure to surgical disciplines for pre-clinical medical students.

\section{Acknowledgements}

We acknowledge the Meharry Medical College Pamela C. Williams Simulation \& Clinical Skills Center.

\section{Conflict of interest}

Jordan Baechle reports having a patent pending for the design of the laparoscopic simulator box used in this study under the trademark SurgeonBox.

\section{References}

1. Garner MS, Gusberg RJ, Kim AW. The positive effect of immediate feedback on medical student education during the surgical clerkship. J Surg Educ 2014; 71: 391-397. https://doi.org/10.1016/j.jsurg.2013.10.009.

2. Ladak A, Hanson J, de Gara CJ. What procedures are students doing during undergraduate surgical clerkship? Can J Surg 2006; 49: 329-334..

3. Debas HT, Bass BL, Brennan MF, Flynn TC, Folse RJ, Freischlag JA, et al. American Surgical Association Blue Ribbon Committee report on surgical education: 2004. Ann Surg 2005; 241: 1-8. https://doi.org/10.1097/01.sla.0000150066. 83563.52 .

4. Bernholt DL, Garzon-Muvdi J, LaPorte DM, Yang SC, McFarland EG. A survey of current policy and practice of surgical exposure for preclerkship medical students at American medical institutions. Am J Surg 2013; 206: 433438. https://doi.org/10.1016/j.amjsurg.2013.01.032. 
5. Gauvin JM. How to promote medical students' interest in surgery. Surgery 2003; 134: 407-408. https://doi.org/10. 1067/s0039-6060(03)00110-7.

6. Glasgow SC, Tiemann D, Frisella MM, Conroy G, Klingensmith M. Laparoscopy as an educational and recruiting tool. Am J Surg 2006; 191: 542-544. https://doi.org/10. 1016/j.amjsurg.2006.09.033.

7. Salna M, Sia T, Curtis G, Leddy D, Widmann WD. Sustained increased entry of medical students into surgical careers: a student-led approach. J Surg Educ 2016; 73: 151-156. https://doi.org/10.1016/j.jsurg.2015.08.012.

8. Riboh J, Curet $M$, Krummel $T$. Innovative introduction to surgery in the preclinical years. Am J Surg 2007; 194: 227230. https://doi.org/10.1016/j.amjsurg.2006.12.038.

9. Miller S, Shipper E, Hasty B, Merrell SB, Lee EW, Lin D, et al. Introductory surgical skills course: technical training and preparation for the surgical environment. MedEdPORTAL 2018; 14: 10775. https://doi.org/10.15766/mep_2374-8265.10775.

10. McKinley SK, Kochis M, Cooper CM, Saillant N, Haynes AB, Petrusa E, et al. Medical students' perceptions and motivations prior to their surgery clerkship. Am J Surg 2019; 218: 424-429. https://doi.org/10.1016/j.amjsurg.2019.01.010.

11. Kozar RA, Lucci A, Miller CC, Azizzadeh A, Cocanour CS, Potts JR, et al. Brief intervention by surgeons can influence students toward a career in surgery. J Surg Res 2003; 111: 166-169. https://doi.org/10.1016/s0022-4804(03)00104-5.

12. Thampy H, Nicola K. Peer-assisted learning for foundation doctors. Clin Teach 2017; 14: 180-183. https://doi.org/10. $1111 /$ tct.12586.

13. Silbert BI, Lake FR. Peer-assisted learning in teaching clinical examination to junior medical students. Med Teach 2012; 34 : 392-397. https://doi.org/10.3109/0142159x.2012.668240.

14. Wagner M, Mileder LP, Goeral K, Klebermass-Schrehof K, Cardona FS, Berger A, et al. Student peer teaching in paediatric simulation training is a feasible low-cost alternative for education. Acta Paediatr 2017; 106: 995-1000. https://doi. org/10.1111/apa.13792.

15. Hughes TC, Jiwaji Z, Lally K, Lloyd-Lavery A, Lota A, Dale A, et al. Advanced Cardiac Resuscitation Evaluation (ACRE): a randomised single-blind controlled trial of peer-led vs. expertled advanced resuscitation training. Scand J Trauma Resusc Emerg Med 2010; 18: 3. https://doi.org/10.1186/ 1757-7241-18-3.

16. House JB, Choe CH, Wourman HL, Berg KM, Fischer JP, Santen SA. Efficient and effective use of peer teaching for medical student simulation. West J Emerg Med 2017; 18: 137-141. https://doi.org/10.5811/westjem.2016.11.32753.

17. Heckmann JG, Dütsch M, Rauch C, Lang C, Weih M, Schwab S. Effects of peer-assisted training during the neurology clerkship: a randomized controlled study. Eur J Neurol 2008; 15: 1365-1370. https://doi.org/10.1111/j.1468-1331.2008.02317.x.
18. Knobe M, Münker R, Sellei RM, Holschen M, Mooij SC, Schmidt-Rohlfing B, et al. Peer teaching: a randomised controlled trial using student-teachers to teach musculoskeletal ultrasound. Med Educ 2010; 44: 148-155. https://doi.org/10. 1111/j.1365-2923.2009.03557.x.

19. Agius A, Stabile I. Undergraduate peer assisted learning tutors' performance in summative anatomy examinations: a pilot study. Int J Med Educ 2018; 9: 93-98. https://doi. org/10.5116/ijme.5aa3.e2a6.

20. Bennett SR, Morris SR, Mirza S. Medical students teaching medical students surgical skills: the benefits of peer-assisted learning. J Surg Educ 2018; 75: 1471-1474. https://doi.org/10. 1016/j.jsurg.2018.03.011.

21. Peters JH, Fried GM, Swanstrom LL, Soper NJ, Sillin LF, Schrimer B, et al. Development and validation of a comprehensive program of education and assessment of the basic fundamentals of laparoscopic surgery. Surgery 2004; 135: 21-27. https://doi.org/10.1016/s0039-6060(03)00156-9.

22. Madan AK, Frantzides CT, Park WC, Tebbit CL, Kumari NV, O'Leary PJ. Predicting baseline laparoscopic surgery skills. Surg Endosc 2005; 19: 101-104. https://doi.org/10. 1007/s00464-004-8123-7.

23. Fraser SA, Feldman LS, Stanbridge D, Fried GM. Characterizing the learning curve for a basic laparoscopic drill. Surg Endosc 2005; 19: 1572-1578. https://doi.org/10. 1007/s00464-005-0150-5.

24. Galiñanes EL, Shirshenkan JR, Doty J, Wakefield MR, Ramaswamy A. Standardized laparoscopic simulation positively affects a student's surgical experience. J Surg Educ 2013; 70: 508-513. https://doi.org/10.1016/j.jsurg.2013.03.005.

25. Xeroulis GJ, Park J, Moulton CA, Reznick RK, Leblanc V, Dubrowski A. Teaching suturing and knot-tying skills to medical students: a randomized controlled study comparing computer-based video instruction and (concurrent and summary) expert feedback. Surgery 2007; 141: 442-449. https://doi. org/10.1016/j.surg.2006.09.012.

26. Antiel RM, Thompson SM, Camp CL, Thompson GB, Farley DR. Attracting students to surgical careers: preclinical surgical experience. J Surg Educ 2012; 69: 301-305. https://doi.org/10. 1016/j.jsurg.2011.10.001.

27. Sanders CW, Edwards JC, Burdenski TK. A survey of basic technical skills of medical students. Acad Med 2004; 79: 873875. https://doi.org/10.1097/00001888-200409000-00013.

28. Patel MS, Mowlds DS, Khalsa B, Foe-Parker JE, Rama A, Jafari F, et al. Early intervention to promote medical student interest in surgery and the surgical subspecialties. J Surg Educ 2013; 70: 81-86. https://doi.org/10.1016/j.jsurg.2012.09.001.

29. Sant'Ana GM, Cavalini W, Negrello B, Bonin EA, Dimbarre $\mathrm{D}$, Claus $\mathrm{C}$, et al. Retention of laparoscopic skills in naive medical students who underwent short training. Surg Endosc 2017; 31: 937-944. https://doi.org/10.1007/ s00464-016-5063-y. 
30. Abbas P, Holder-Haynes J, Taylor DJ, Scott BG, Brandt ML, Naik-Mathuria B. More than a camera holder: teaching surgical skills to medical students. J Surg Res 2015; 195: 385-389. https://doi.org/10.1016/j.jss.2015.01.035.

31. Brundage SI, Lucci A, Miller CC, Azizzadeh A, Spain DA, Kozar RA. Potential targets to encourage a surgical career. J Am Coll Surg 2005; 200: 946-953. https://doi.org/10.1016/j. jamcollsurg.2005.02.033.

32. Giantini Larsen AM, Pories S, Parangi S, Robertson FC. Barriers to pursuing a career in surgery: an institutional survey of Harvard Medical School students. Ann Surg 2019. https://doi.org/10.1097/SLA.0000000000003618.

33. Marks IH, Diaz A, Keem M, Ladi-Seyedian Seyedeh-Sanam, Philipo GS, Munir GS, et al. Barriers to women entering surgical careers: a global study into medical student perceptions. World J Surg 2020; 44: 37-44. https://doi.org/10. 1007/s00268-019-05199-1.

34. Walker NR, Deekonda P, Glasbey JC, Rashid S, Gokani VJ, Humm G, et al. Attracting medical students and doctors into surgical training in the UK and Ireland. Int J Surg 2019; 67: 107-112. https://doi.org/10.1016/j.ijsu.2019.01.007.

35. Capers 4th Q, Clinchot D, McDougle L, Greenwald AG. Implicit racial bias in medical school admissions. Acad Med 2017; 92: 365-369. https://doi.org/10.1097/ACM.000000000 0001388 .

36. Andriole DA, Jeffe DB, Schechtman KB. Is surgical workforce diversity increasing? J Am Coll Surg 2007; 204: 469-477. https://doi.org/10.1016/j.jamcollsurg.2006.12.035.

37. Blakemore LC, Hall JM, Biermann JS. Women in surgical residency training programs. J Bone Joint Surg Am 2003; 85: 2477-2480. https://doi.org/10.2106/00004623-20031200000031 .

\section{Appendix: laparoscopic task outline}

\section{Task one: ball transfer}

Equipment: Two Maryland forceps, six cups, 15 balls

Arrange three tall plastic cups (a) on points \#1-3 provided on the Ball Transfer Backdrop and three short cups (b) with five balls in each within the center circle. Balls transferred to cup \#1 must be transferred with the left instrument while those transferred to \#3 must be transferred with the right instrument. \#2 cup may receive balls carried by either instrument. The short cups (b) should remain within the black circle and the tall cups (a) must remain on top of the specified zone.

\section{Task two: precision cutting}

Equipment: One Maryland forceps, one endoscopic scissors, one $4 \times 4$ Precision Cutting Backdrop printout

After cutting along the dotted lines, secure the posterior Precision Cutting Backdrop printout against the cardboard backdrop. The participant will attempt to cut out the circle along the black line without deviating from printed black line. The timer will start when the tools enter the operating field and stop when the circle has been completely sectioned from the original cut out.

\section{Task three: zip tie ligation loop}

Equipment: Two Maryland forceps

In this task, the participant will arrange and secure a ligation loop around an inflated latex glove appendage using a zip tie. The zip tie may be pre-loaded in the tool before insertion. The time will start once a tool enters the operating field.

\section{Task four: precision beading}

Equipment: Two Maryland forceps, one $10-\mathrm{cm}$ suture (knotted on one end), five beads, one cup

The participant will be given a $10-\mathrm{cm}$ needled suture, knotted on the opposite end of the needle so that the beads may not pass through. The needle may be preloaded before inserting the forceps and starting the timer. The five beads will be centered in the operating field within a cup. The participant may orient the tool in their preferred ports.

\section{Task five: suture knotting}

Equipment: Two Maryland forceps, optional needle driver, one $10-\mathrm{cm}$ suture, suture pad or Penrose drain

For this task the participant will place a short suture through the two marks and tie three throws of a knot intracorporeally, in order to close the slit provided. The first throw should be a surgeon's knot, followed by two single throws. The needle should exchange hands, or the needle end of the suture, between each throw to ensure each throw is with the opposite hand. 